\title{
CONTROLE Químico DE Pistia stratiotes, Eichhornia crassipes E Salvinia molesta EM CAIXAS D'ÁGUA ${ }^{1}$
}

\author{
Chemical Control of Pistia stratiotes, Eichhornia crassipes and Salvinia molesta in Reservoirs
}

\begin{abstract}
MARTINS, D. ${ }^{2}$, VELINI, E.D. ${ }^{2}$, NEGRISOLI, E. ${ }^{3}$ e TOFOLI, G.R. ${ }^{4}$
RESUMO - Durante o ano de 1999 foram conduzidos três experimentos em Botucatu-SP, em condições de caixas d'água, com o objetivo de estudar o efeito de alguns herbicidas sobre Pistia stratiotes, Eichhornia crassipes e Salvinia molesta. Os herbicidas e as doses utilizadas foram: diquat a 460, 960 e $1.400 \mathrm{~g} \mathrm{ha}^{-1} \mathrm{com}$ e sem o surfatante Agral a 0,1\%; 2,4 D a 1.340; glyphosate a $3.360 \mathrm{~g} \mathrm{ha}^{-1}$ mais $0,5 \%$ do surfatante Aterbane; e imazapyr a $250 \mathrm{~g} \mathrm{ha}^{-1}$, além de uma testemunha sem aplicação de herbicida. Os estudos foram instalados no delineamento experimental em blocos ao acaso, com quatro repetições. As parcelas experimentais foram constituídas por caixas d'água com dimensões de $60 \times 60 \times 60 \mathrm{~cm}$ mantidas a pleno sol no campo. Utilizaram-se 18, 18 e 30 plantas/caixa de P. stratiotes, E. crassipes e S. molesta, respectivamente. Os herbicidas foram aplicados com pulverizador costal a pressão constante de $\mathrm{CO}_{2}$ a 1,75 bar, munido de barra com bicos $110.02 \mathrm{VS}$, com consumo de calda de $180 \mathrm{~L} \mathrm{ha}^{-1}$. O herbicida diquat foi eficiente no controle de S. molesta, P. stratiotes e E. crassipes em todas as doses testadas. Para P. stratiotes, os herbicidas 2,4 D e imazapyr não proporcionaram controle, enquanto o herbicida glyphosate mostrou-se eficiente. Em relação a E. crassipes, os herbicidas $2,4 \mathrm{D}$ e glyphosate foram eficientes em seu controle. Os herbicidas imazapyr, 2,4 D e glyphosate não foram eficientes no controle de S. molesta.
\end{abstract}

Palavras-chave: planta daninha, planta aquática, herbicida.

\begin{abstract}
Three trials were carried out in Brazil in 1999 to study the effect of herbicides on aquatic weed control in reservoirs. The herbicides and doses used were: diquat at 460, 960 and $1,400 \mathrm{~g} \mathrm{ha}^{-1} ; 2.4 \mathrm{D}$ at 1,340 $\mathrm{g} \mathrm{ha}^{-1}$; glyphosate at 3,360 $\mathrm{g} \mathrm{ha}^{-1}$, imazapyr at $250 \mathrm{~g} \mathrm{ha}^{-1}$ and a control. The experiment was arranged in a randomized complete block design with four replications. The plots were constituted by reservoirs with $60 \times 60 \times 60 \mathrm{~cm}$ dimension. The diquat herbicide at all doses showed excellent control of Salvinia molesta, Pistia stratiotes and Eichhornia crassipes. The herbicides $2.4 \mathrm{D}$ and imazapyr did not promote a good control for $\boldsymbol{P}$. stratiotes while glyphosate showed a very good control. For E. crassipes, $2.4 \mathrm{D}$ and glyphosate showed an efficient control. The herbicides imazapyr, 2.4 D and glyphosate did not show a good control over S. molesta.
\end{abstract}

Key words: weed, aquatic plant, herbicide.

\section{INTRODUÇÃO}

Uma grande diversidade de plantas aquáticas pode ser encontrada vegetando as margens de rios e reservatórios ou dentro dos mais diversos ambientes aquáticos, empregando diferentes mecanismos de adaptação para sobrevivência e desenvolvimento. Enquanto algumas espécies apresentam-se enraizadas em corpos d'água com fortes correntezas, outras somente podem viver em águas paradas ou estagnadas.

Recebido para publicação em 5/11/2001 e na forma revisada em 18/12/2001.

2 Professores Assistentes Doutores, Departamento de Produção Vegetal da FCA-UNESP, Caixa Postal 237, 18603-970 BotucatuSP, <dago@ fca.unesp.br>; ${ }^{3}$ Eng.-Agr., aluno de Pós-Graduação em Agricultura em nível de Mestrado, Departamento de Produção Vegetal da FCA-UNESP; ${ }^{4}$ Eng.-Agr., aluno de Pós-Graduação em Proteção de Plantas em nível de Doutorado, Departamento de Produção Vegetal da FCA-UNESP. 
As plantas aquáticas que proliferam de forma desorganizada nos reservatórios e rios podem provocar vários problemas, como: acúmulo de lixo e outros sedimentos, proliferação de vetores de doenças, dificuldades na navegação, prejuízos ao turismo e à pesca; nestes casos, podem ser denominadas daninhas. Essas plantas afetam ainda o transporte de cargas das hidrovias, dificultando as rotas de navegação, além de determinados prejuízos às usinas hidrelétricas na geração de energia, o que incrementa os custos de manutenção e geração (Marcondes \& Tanaka, 1997).

Dentre as principais plantas daninhas aquáticas, podem-se destacar: Eichhornia crassipes, Pistia stratiotes, Echinochloa polystachya; espécies aquáticas dos gêneros Polygonum e Salvinia; Brachiaria subquadripara; Typha dominguensis; Egeria densa; Egeria najas; e espécies aquáticas do gênero Cyperus.

As plantas daninhas aquáticas flutuantes são as que causam os mais sérios e difundidos problemas em nível mundial. Elas normalmente apresentam rápida capacidade de multiplicação vegetativa, independência das estruturas sexuais de reprodução, grande área de tecido fotossintético em proporção ao comprimento da planta, capacidade rápida de ocupar locais disponíveis onde incida luz, além da independência das condições do substrato, devido ao fluxo d'água e à localização das plantas (IBAMA, 1998).

E. crassipes (aguapé) é uma planta aquática flutuante livre, nativa da América do Sul e pertencente à família Pontederidaceae, que se reproduz por sementes, as quais permanecem viáveis por pelo menos 15 anos no sedimento dos corpos d'água (Holm \& Yeo, 1980), e de forma vegetativa, via estolões. Atualmente, encontra-se amplamente distribuída nas regiões tropicais e subtropicais.

P. stratiotes (alface-d'água), pertencente à família Araceae, é uma espécie flutuante que apresenta capacidade de rápida multiplicação vegetativa, habilidade para regenerar-se a partir de pequenas porções do talo e independência parcial ou completa das estruturas sexuais de reprodução.

Salvinia molesta (salvínia) é uma espécie da família Salviniaceae que apresenta ampla distribuição no Brasil, constituindo-se em sério problema como invasora em diferentes coleções de águas. É uma planta muito comum em mananciais de águas paradas e ricas em nutrientes. Sob condições ótimas, forma uma enorme massa vegetativa na superfície das águas e chega a produzir 650 gramas de biomassa seca $\mathrm{m}^{-2}$ ano $^{-1}$. A sua decomposição pode levar ao esgotamento do oxigênio dissolvido na água, e a sua cobertura superficial pode impedir o arejamento e a penetração de luz.

O objetivo deste trabalho foi o de avaliar diferentes herbicidas e doses no controle de plantas de $P$. stratiotes, E. crassipes e $S$. molesta, em caixa d'água.

\section{MATERIAL E MÉTODOS}

Foram conduzidos três experimentos com plantas daninhas aquáticas na Faculdade de Ciências Agronômicas/UNESP, campus de Botucatu-SP, no núcleo de Pesquisas Avançadas em Matologia.

As plantas daninhas aquáticas estudadas foram $P$. stratiotes, E. crassipes e S. molesta, as quais foram cultivadas em caixas de fibra de vidro de $60 \times 60 \times 60 \mathrm{~cm}$, com 120 litros de água. Foi colocada em cada caixa uma camada de solo de 20 litros. As características químicas do solo utilizado nos diferentes experimentos foram: $\mathrm{pH}\left(\mathrm{CaCl}_{2}\right)=4,5$; M.O. $\left(\mathrm{g} \mathrm{dm}^{-3}\right)=27$; , $\mathrm{Cu}, \mathrm{Fe}, \mathrm{Mn}$ e $\mathrm{Zn}=10,0,26,8,26,48,6$ e $0,9 \mathrm{mg} \mathrm{dm}^{-3}$, respectivamente; $\mathrm{H}+\mathrm{Al}, \mathrm{K}, \mathrm{Ca}, \mathrm{Mg}$, $\mathrm{SB}$ e CTC $=52,2,3,20,8,30$ e $82 \mathrm{mmol}_{\mathrm{c}} \mathrm{dm}^{-3}$, respectivamente; e V\% $=37$. As características físicas do solo eram: $60 \%$ de argila, 34\% de areia e $6 \%$ de silte. Cada caixa foi adubada com 40 g da fórmula 4-14-8 de NPK.

As caixas d'água foram mantidas em pleno sol, sendo utilizadas 18, 18 e 30 plantas/caixa de $P$. stratiotes, E. crassipes e S. molesta, respectivamente. Os tratamentos foram dispostos no delineamento experimental em blocos ao acaso, com quatro repetições.

Os tratamentos utilizados nos três experimentos com as diferentes espécies foram: diquat (Reward) a 460, 960 e $1.400 \mathrm{~g} \mathrm{ha}^{-1}$, com e sem o surfatante Agral a 0,1\%;2,4 D (U 46 D Fuid) a $1.340 \mathrm{~g} \mathrm{ha}^{-1}$; glyphosate (Rodeo) a $3.360 \mathrm{~g} \mathrm{ha}^{-1}+0,5 \%$ de Aterbane; e imazapyr 
(Arsenal) $250 \mathrm{~g} \mathrm{ha}^{-1}$. Houve, ainda, uma testemunha sem aplicação de herbicidas. Os herbicidas foram aplicados em plantas adultas, coletadas no reservatório de Barra Bonita-SP, sendo as plantas distribuídas uniformemente nas caixas d'água.

A aplicação dos herbicidas foi feita com um pulverizador costal a pressão constante de $\mathrm{CO}_{2}$ a 1,75 bar. O consumo de calda utilizado foi de $180 \mathrm{~L} \mathrm{ha}^{-1}$. A barra de aplicação apresentava-se com dois bicos de jato plano, tipo TeeJet $110.02 \mathrm{VS}$, distanciados entre si de $0,5 \mathrm{~m}$.

As condições ambientais durante a aplicação dos herbicidas estão apresentadas na Tabela 1, tendo sido divididas em condição inicial, intermediária e final, durante o tempo em que se realizou a aplicação dos herbicidas, que foi de 50 minutos para os experimentos de $P$. stratiotes e E. crassipes e de 35 minutos para o de $S$. molesta.

Tabela 1 - Condições ambientais durante a aplicação dos herbicidas para os diferentes experimentos. BotucatuSP, 1999

\begin{tabular}{|l|c|c|c|}
\hline \multicolumn{1}{|c|}{ Condição } & $\begin{array}{c}\text { Temperatura } \\
\left({ }^{\circ} \mathrm{C}\right)\end{array}$ & $\begin{array}{c}\text { Umidade } \\
\text { relativa }(\%)\end{array}$ & $\begin{array}{c}\text { Velocidade do } \\
\text { vento }\left(\mathrm{m} \mathrm{s}^{-1}\right)\end{array}$ \\
\hline \multicolumn{3}{|c|}{ Experimentos com P. stratiotes e E. crassipes } \\
\hline Inicial & 28 & 78 & 0,0 \\
Intermediária & 27 & 76 & 0,0 \\
Final & 28 & 78 & 0,0 \\
\hline \multicolumn{4}{|c|}{ Experimento com S. molesta } \\
\hline Inicial & 26 & 72 & 0,5 \\
Intermediária & 26 & 72 & 0,4 \\
Final & 26 & 72 & 0,5 \\
\hline
\end{tabular}

As plantas foram avaliadas visualmente aos 2, 5, 8, 10, 15, 22 e 29 dias após a aplicação (DAA), por meio de uma escala percentual de notas, em que zero representava nenhum controle e $100 \%$, controle total das plantas para a espécie $P$. stratiotes; aos 5, 7, 9, 11, 16 e 20 DAA para E. crassipe; e aos 5, 7, 9, 11, 16, 20, 22, 26 e 32 DAA para S. molesta. As avaliações foram realizadas até desaparecimento ou estabilização dos sintomas de fitointoxicação (Sociedade ...SBCPD, 1995).

Os parâmetros utilizados para o estabelecimento das notas foram: quantidade de plantas mortas, acúmulo de biomassa, inibição do crescimento, quantidade e uniformidade das injúrias e capacidade de rebrota das plantas.

Os resultados obtidos foram submetidos à análise de variância pelo teste $\mathrm{F}$, sendo as médias dos tratamentos comparadas pelo teste t a $5 \%$ de probabilidade.

\section{RESULTADOS E DISCUSSÃO}

\section{Experimento com Pistia stratiotes}

Nas caixas d'água em que se aplicou o herbicida glyphosate, os sintomas visuais e iniciais foram amarelecimento das folhas, seguido de queimaduras e necroses. Para o herbicida 2,4 D, o sintoma inicial foi encarquilhamento inicial das folhas de alface-d'água, mantendo-se os sintomas até o final do experimento. O diquat promoveu queimaduras severas nas folhas, evoluindo para necrose e, em seguida, morte das plantas.

Na Tabela 2 estão apresentados os resultados da avaliação de controle sobre as plantas de $P$. stratiotes em diferentes épocas após a aplicação dos herbicidas. Nota-se, aos 2 dias após a aplicação (DAA) dos tratamentos químicos, que somente o herbicida diquat proporcionou controle considerado bom a excelente das plantas de alface-d'água mesmo nas doses menores (460 $\mathrm{g} \mathrm{ha}^{-1}$ ), independentemente da adição do surfatante Agral. Aos 8 DAA dos herbicidas, todas as doses de diquat testadas proporcionaram a morte completa das plantas em estudo. Os mesmos resultados observados por MacDonald \& Langeland (2001) foram semelhantes aos aqui encontrados, no qual o herbicida diquat proporcionou excelente controle sobre várias espécies de plantas aquáticas, como Lemna spp., Azolla caroliniana, E. crassipes, além de $P$. stratiotes. Os pesquisadores relatam também que o herbicida diquat proporcionou excelente controle de espécies submersas, como: Ceratofhyllum demersum, Hydrilla vereticillata, Pontamogetun spp., Utricularia spp. e Cabomba caroliniana.

No que se refere à utilização do herbicida glyphosate, aos cinco dias após a aplicação o composto apresentou controle considerado bom, e, a partir dos 10 DAA, já era excelente; no entanto, deve ser ressaltado que houve 
rebrota de uma planta em uma das repetições desse tratamento. Resultado similar foi encontrado por Martins et al. (1999), os quais observaram que, de todos os herbicidas testados no estudo, apenas o glyphosate aos 7 DAA apresentava controle eficiente das plantas de alface-d'água.

Os herbicidas imazapyr e 2,4 D proporcionaram injúrias às plantas de $P$. stratiotes, porém não com intensidade que possa ser considerada como controle, durante o período de condução do experimento. Houve rebrotas das plantas em todas as parcelas.

\section{Experimento com Salvinia molesta}

Na Tabela 3 estão apresentados os resultados das avaliações de controle sobre plantas de S. molesta em diferentes épocas após a aplicação dos herbicidas. Observa-se que os sintomas de intoxicação iniciais nas plantas de salvínia foram mais severos já no início dos estudos, aos 5 DAA, com a aplicação de diquat, independentemente da dose testada. O controle proporcionado pelo herbicida diquat foi incrementado no decorrer do tempo e, aos 9 DAA, as diferentes doses de diquat proporcionaram controles que variam de bom a excelente. Verificou-se, também, que nessa ocasião os herbicidas
$2,4 \mathrm{D}$, glyphosate e imazapyr causaram poucas injúrias às plantas em estudo, com os primeiros sintomas aparecendo apenas aos 7 DAA.

A partir dos 16 DAA, as diferentes doses de diquat testadas proporcionaram controles excelentes (acima de 94\%), que se mantiveram elevados até o final das avaliações. A adição de surfatante Agral à calda de aplicação de diquat não incrementou o controle de S. molesta, em comparação aos tratamentos sem sua utilização, em nenhuma das épocas de avaliação.

Ressalta-se que os herbicidas $2,4 \mathrm{D}$ e imazapyr mostraram-se quase inócuos no controle desta espécie e o herbicida glyphosate proporcionou controle insatisfatório durante as avaliações conduzidas. Em um outro estudo, também Martins et al. (1999) verificaram que os herbicidas imazapyr, 2,4 D e glyphosate não foram eficientes no controle de S. molesta, em todas as doses testadas na ocasião.

\section{Experimento com Eichhornia crassipes}

Os primeiros sintomas de toxicidade nas plantas de E. crassipes observados para o herbicida glyphosate foram amarelecimento das folhas, seguido de queimaduras e necroses, imergindo após o final do estudo.

Tabela 2 - Porcentagem de controle de Pistia stratiotes aos 2, 5, 8, 10, 15, 22 e 29 dias após aplicação dos herbicidas. Botucatu-SP, 1999

\begin{tabular}{|c|c|c|c|c|c|c|c|c|}
\hline \multirow{2}{*}{ Tratamento } & \multirow{2}{*}{$\begin{array}{c}\text { Dose } \\
\left(\mathrm{g} \mathrm{i.a.} \mathrm{ha}^{-1}\right)\end{array}$} & \multicolumn{7}{|c|}{ Dias após a aplicação } \\
\hline & & 2 & 5 & 8 & 10 & 15 & 22 & 29 \\
\hline 1. diquat & 460 & $97,2 \mathrm{a}$ & $97,2 \mathrm{~b}$ & $100,0 \mathrm{a}$ & $100,0 \mathrm{a}$ & $100,0 \mathrm{a}$ & $100,0 \mathrm{a}$ & $100,0 \mathrm{a}$ \\
\hline 2. diquat ${ }^{1 /}$ & 460 & $97,7 \mathrm{a}$ & $100,0 \mathrm{a}$ & $100,0 \mathrm{a}$ & $100,0 \mathrm{a}$ & $100,0 \mathrm{a}$ & $100,0 \mathrm{a}$ & $100,0 \mathrm{a}$ \\
\hline 3. diquat & 960 & 99,0 a & $100,0 \mathrm{a}$ & $100,0 \mathrm{a}$ & $100,0 \mathrm{a}$ & $100,0 \mathrm{a}$ & $100,0 \mathrm{a}$ & $100,0 \mathrm{a}$ \\
\hline 4. diquat ${ }^{1 /}$ & 960 & $97,5 \mathrm{a}$ & $100,0 \mathrm{a}$ & $100,0 \mathrm{a}$ & $100,0 \mathrm{a}$ & $100,0 \mathrm{a}$ & $100,0 \mathrm{a}$ & $100,0 \mathrm{a}$ \\
\hline 5. diquat & 1.400 & $99,0 \mathrm{a}$ & $100,0 \mathrm{a}$ & $100,0 \mathrm{a}$ & $100,0 \mathrm{a}$ & $100,0 \mathrm{a}$ & $100,0 \mathrm{a}$ & $100,0 \mathrm{a}$ \\
\hline 6. diquat ${ }^{1 /}$ & 1.400 & 99,0 a & $72,5 \mathrm{~d}$ & $100,0 \mathrm{a}$ & $100,0 \mathrm{a}$ & $100,0 \mathrm{a}$ & $100,0 \mathrm{a}$ & $100,0 \mathrm{a}$ \\
\hline 8. glyphosate ${ }^{2,3 /}$ & 3.336 & $22,0 \mathrm{~b}$ & $87,0 \mathrm{c}$ & $91,5 \mathrm{~b}$ & $99,2 \mathrm{a}$ & $99,5 \mathrm{a}$ & $99,5 \mathrm{a}$ & 99,5 a \\
\hline 7. $2,4-\mathrm{D}^{2 /}$ & 1.340 & $6,7 \mathrm{c}$ & $12,0 \mathrm{f}$ & $12,5 \mathrm{c}$ & $16,2 \mathrm{~b}$ & $16,5 \mathrm{c}$ & $21,2 \mathrm{~b}$ & $21,2 \mathrm{~b}$ \\
\hline 9. imazapyr & 250 & $4,0 \mathrm{c}$ & $14,5 \mathrm{e}$ & $13,2 \mathrm{c}$ & $16,0 \mathrm{~b}$ & $21,2 \mathrm{~b}$ & $16,7 \mathrm{~b}$ & $16,7 \mathrm{~b}$ \\
\hline 10. testemunha & - & - & - & - & - & - & - & - \\
\hline F tratamentos & & $573,54 * *$ & $1957,64 * *$ & $691,82 * *$ & $1329,00 * *$ & $500,88 * *$ & $492,70 * *$ & $492,70 * *$ \\
\hline F bloco & & $2,43^{\mathrm{ns}}$ & $1,30^{\mathrm{ns}}$ & $1,38^{\mathrm{ns}}$ & $1,31^{\mathrm{ns}}$ & $1,23^{\mathrm{ns}}$ & $1,16^{\mathrm{ns}}$ & $1,16^{\mathrm{ns}}$ \\
\hline $\mathrm{CV}(\%)$ & & 5,3 & 2,1 & 3,6 & 2,5 & 3,9 & 3,9 & 3,9 \\
\hline d.m.s. & & 5,35 & 2,42 & 4,21 & 2,99 & 4,63 & 4,69 & 4,69 \\
\hline
\end{tabular}

${ }^{1 /}$ Foi adicionado Agral a $0,1 \% ;{ }^{2}$ dose em equivalente ácido por ha; $\mathrm{e}^{\underline{3} /}$ foi adicionado Aterbane a $0,5 \%$.

Médias seguidas de mesma letra, na coluna, não diferem estatisticamente entre si pelo teste $\mathrm{t}(\mathrm{P}>0,05)$. 
Tabela 3 - Porcentagem de controle de Salvinia molesta aos 5, 7, 9, 11, 16, 20, 26 e 32 dias após a aplicação dos herbicidas. Botucatu-SP, 1999

\begin{tabular}{|c|c|c|c|c|c|c|c|c|c|}
\hline \multirow{2}{*}{ Tratamento } & \multirow{2}{*}{$\begin{array}{c}\text { Dose } \\
\left(\mathrm{g} \mathrm{i.a.} \mathrm{ha}^{-1}\right)\end{array}$} & \multicolumn{8}{|c|}{ Dias após a aplicação } \\
\hline & & 05 & 07 & 09 & 11 & 16 & 20 & 26 & 32 \\
\hline 1. diquat & 960 & $35,00 \mathrm{~b}$ & $71,25 \mathrm{a}$ & 86,25 a & $92,75 \mathrm{~b}$ & $98,75 \mathrm{a}$ & $99,75 \mathrm{a}$ & 99,75 a & 99,75 a \\
\hline 2. diquat ${ }^{1 /}$ & 960 & $21,75 \mathrm{c}$ & $72,75 \mathrm{a}$ & $83,25 \mathrm{a}$ & $91,75 \mathrm{~b}$ & $94,75 \mathrm{a}$ & 95,25 a & $97,75 \mathrm{a}$ & 98,25 a \\
\hline 3. diquat & 1400 & $45,00 \mathrm{a}$ & $78,25 \mathrm{a}$ & 90,00 a & $95,00 \mathrm{ab}$ & $99,25 \mathrm{a}$ & $100,00 \mathrm{a}$ & $100,00 \mathrm{a}$ & $100,00 \mathrm{a}$ \\
\hline 4. diquat ${ }^{1 /}$ & 1400 & $36,25 \mathrm{ab}$ & $80,00 \mathrm{a}$ & 98,25 a & $97,25 \mathrm{a}$ & 99,75 a & $99,75 \mathrm{a}$ & $100,00 \mathrm{a}$ & $100,00 \mathrm{a}$ \\
\hline 5. $2,4-D^{2 /}$ & 1340 & $0,00 \mathrm{~d}$ & $2,25 \mathrm{~b}$ & $4,00 \mathrm{~b}$ & $7,50 \mathrm{~d}$ & $1,00 \mathrm{~d}$ & $0,00 \mathrm{~d}$ & $0,00 \mathrm{~d}$ & $0,00 \mathrm{c}$ \\
\hline 6. glyphosate $e^{2,3 /}$ & 3360 & $0,00 \mathrm{~d}$ & $0,75 \mathrm{~b}$ & $3,75 \mathrm{~b}$ & $17,75 \mathrm{c}$ & $48,75 \mathrm{~b}$ & $65,00 \mathrm{~b}$ & $52,5 \mathrm{~b}$ & $53,75 \mathrm{~b}$ \\
\hline 7. imazapyr & 250 & $0,00 \mathrm{~d}$ & $1,50 \mathrm{~b}$ & $4,75 \mathrm{~b}$ & $6,75 \mathrm{~d}$ & $8,75 \mathrm{c}$ & $15,50 \mathrm{c}$ & $9,50 \mathrm{c}$ & $2,50 \mathrm{c}$ \\
\hline 8. testemunha & - & - & - & - & - & - & - & - & - \\
\hline F tratamento & & $39,15^{*}$ & $137,50 *$ & $328,60^{*}$ & $1.147,10^{*}$ & $442,60^{*}$ & $368,00^{*}$ & $715,20^{*}$ & $2.095,30 *$ \\
\hline F bloco & & $0,21^{\mathrm{ns}}$ & $0,51^{\mathrm{ns}}$ & $0,43^{\mathrm{ns}}$ & $3,27^{\mathrm{ns}}$ & $1,47^{\mathrm{ns}}$ & $1,23^{\mathrm{ns}}$ & $2,44^{\mathrm{ns}}$ & $1,99^{\mathrm{ns}}$ \\
\hline $\mathrm{CV}(\%)$ & & 31,85 & 15,45 & 9,48 & 4,53 & 6,57 & 6,62 & 5,13 & 3,13 \\
\hline d.m.s. & & 9,33 & 10,05 & 7,28 & 3,93 & 6,29 & 6,68 & 5,00 & 3,02 \\
\hline
\end{tabular}

${ }^{1 /}$ Foi adicionado Agral a $0,1 \% ;{ }^{2 /}$ dose em equivalente ácido por ha; $\mathrm{e}^{\underline{3} /}$ foi adicionado Aterbane a $0,5 \%$.

Médias seguidas de mesma letra, na coluna, não diferem estatisticamente entre si pelo teste t $(\mathrm{P}>0,05)$.

Para o herbicida 2,4 D, o sintoma inicial foi um encarquilhamento inicial das folhas de aguapé, que evoluíram para amarelecimento e necroses, com posterior afundamento na lâmina de água.

O herbicida diquat proporcionou queimaduras severas nas folhas, evoluindo posteriormente para necrose e, em seguida, morte da planta. É importante ressaltar que as plantas pulverizadas com diquat, após a sua morte, ficaram flutuando nas caixas por pelo menos 60 dias.

Na Tabela 4 estão apresentados os resultados das avaliações de controle sobre as plantas de E. crassipes em diferentes épocas após a aplicação dos herbicidas. Aos 5 DAA, somente o diquat proporcionou controle considerado satisfatório das plantas de aguapé, com exceção da dose menor (460 $\mathrm{g} \mathrm{ha}^{-1}$ ), independentemente da adição do surfatante Agral.

O controle das plantas evoluiu no decorrer do período experimental, e aos 9 DAA o diquat apresentou controles que variaram de bom a excelente, dependendo da dose testada. Nessa época, notaram-se ainda incrementos na porcentagem de controle proporcionado pelos herbicidas 2,4 D e glyphosate, porém insatisfatórios.

Aos 11 e 16 DAA, verifica-se que a porcentagem de controle das plantas de aguapé proporcionada pelas diferentes doses de diquat foi bem semelhante, variando de bom a excelente. Deve-se destacar que nesses períodos ocorreram grandes incrementos no controle das plantas de aguapé com a utilização de glyphosate e 2,4 D, com destaque para o 2,4 D, que atingiu controles considerados excelentes já aos 11 dias. Também Cardoso (2001), ao estudar a sensibilidade de diferentes acessos de aguapé a herbicidas, coletados em reservatórios do Estado de São Paulo, encontrou para todos os herbicidas testados (imazapyr, glyphosate, diquat e 2,4 D) um controle eficiente das plantas de aguapé, independentemente do genótipo estudado.

Aos 20 DAA, por ocasião do encerramento do experimento, observa-se que todas as doses de diquat foram eficientes no controle das plantas de aguapé, porém a sua menor dose (460 $\mathrm{g} \mathrm{ha}^{-1}$ ), independentemente da adição do surfatante Agral, proporcionou controle inferior ao das maiores doses utilizadas. As doses maiores de diquat (960 e $1.400 \mathrm{~g} \mathrm{ha}^{-1}$ ) causaram morte completa das plantas em estudo.

Os herbicidas $2,4 \mathrm{D}$ e glyphosate, aos 20 DAA, apresentaram controles eficientes sobre as plantas de aguapé, mas ocorreram rebrotas nas caixas tratadas com 2,4 D. Esse resultado corrobora o de Martins et al. (1999), os quais observaram que o herbicida $2,4 \mathrm{D}$ 
Tabela 4 - Porcentagem de controle de E. crassipes aos 5, 7, 9, 11, 16 e 20 dias após aplicação dos herbicidas. Botucatu-SP, 1999

\begin{tabular}{|c|c|c|c|c|c|c|c|}
\hline \multirow{2}{*}{ Tratamento } & \multirow{2}{*}{$\begin{array}{c}\text { Dose } \\
\left(\text { g i.a. ha }{ }^{-1}\right)\end{array}$} & \multicolumn{6}{|c|}{ Dias após a aplicação } \\
\hline & & 05 & 07 & 09 & 11 & 16 & 20 \\
\hline 1. diquat & 460 & $54,75 \mathrm{~b}$ & $71,25 \mathrm{~b}$ & 86,00 a & $94,50 \mathrm{ab}$ & $94,50 \mathrm{bc}$ & $94,50 \mathrm{bc}$ \\
\hline 2. diquat ${ }^{-1}$ & 460 & $56,00 \mathrm{~b}$ & $66,25 \mathrm{~b}$ & 88,75 a & $93,25 \mathrm{~b}$ & $93,25 \mathrm{c}$ & $93,25 \mathrm{c}$ \\
\hline 3. diquat & 960 & $80,00 \mathrm{a}$ & $89,00 \mathrm{a}$ & $96,00 \mathrm{a}$ & $97,75 \mathrm{ab}$ & $98,50 \mathrm{ab}$ & $100,00 \mathrm{a}$ \\
\hline 4. diquat ${ }^{-1}$ & 960 & $80,75 \mathrm{a}$ & $89,50 \mathrm{a}$ & $96,00 \mathrm{a}$ & $98,75 \mathrm{a}$ & $99,50 \mathrm{a}$ & $100,00 \mathrm{a}$ \\
\hline 5. diquat & 1.400 & $80,75 \mathrm{a}$ & $92,25 \mathrm{a}$ & 96,25 a & $98,25 \mathrm{a}$ & $98,75 \mathrm{ab}$ & $100,00 \mathrm{a}$ \\
\hline 6. diquat ${ }^{-1}$ & 1.400 & $82,75 \mathrm{a}$ & $93,00 \mathrm{a}$ & 96,25 a & $98,75 \mathrm{a}$ & $99,25 \mathrm{ab}$ & $100,00 \mathrm{a}$ \\
\hline 7. $2,4-D^{2 /}$ & 3.336 & $5,00 \mathrm{c}$ & $41,75 \mathrm{c}$ & $62,00 \mathrm{~b}$ & $97,50 \mathrm{~b}$ & $98,50 \mathrm{ab}$ & $98,50 \mathrm{ab}$ \\
\hline 8. glyphosate ${ }^{2,3 /}$ & 1.340 & $0,00 \mathrm{c}$ & $23,75 \mathrm{~d}$ & $47,50 \mathrm{c}$ & $58,25 \mathrm{c}$ & $76,75 \mathrm{~d}$ & $100,00 \mathrm{a}$ \\
\hline 9. testemunha & - & - & - & - & - & - & - \\
\hline F tratamentos & & $81,38 * *$ & $254,05 * *$ & $57,35 * *$ & $255,23 * *$ & $226,45^{*} *$ & $246,32 * *$ \\
\hline F bloco & & $0,94^{\mathrm{ns}}$ & $1,07^{\mathrm{ns}}$ & $1,18^{\mathrm{ns}}$ & $0,60^{\mathrm{ns}}$ & $1,05^{\mathrm{ns}}$ & $0,55^{\mathrm{ns}}$ \\
\hline $\mathrm{CV}(\%)$ & & 16,766 & 6,694 & 10,676 & 4,002 & 3,920 & 3,666 \\
\hline d. m. s. & & 11,963 & 6,185 & 11,729 & 4,921 & 4,960 & 4,799 \\
\hline
\end{tabular}

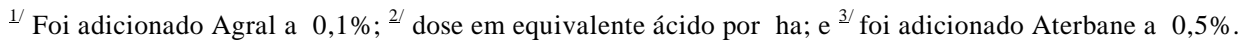

Médias seguidas de mesma letra, na coluna, não diferem estatisticamente entre si pelo teste $\mathrm{t}(\mathrm{P}>0,05)$.

proporcionava bom controle já aos 21 DAA e, aos 28 DAA, um controle excelente e próximo a 100\%. Também Charudattan (1986) observou que os herbicidas 2,4 D e diquat eram eficientes e que o nivel de controle freqüentemente ficava entre 60 e 100\%, podendo ocorrer reinfestações por rebrotas ou por sementes.

\section{LITERATURA CITADA}

CARDOSO, L. R. Sensibilidade de diferentes acessos de aguapé coletados em reservatórios do Estado de São Paulo a herbicidas. Botucatu: Universidade Estadual de São Paulo, 95 p. Dissertação (Mestrado em Ciências Agronômicas) - Universidade Estadual de São Paulo, 2001.

CHARUDATTAN, R. Integrated control of waterhyacinth (Eichhornia crassipes) with a pathogen, insects and herbicides. Weed Sci., v. 34, n. 1, p. 26-30, 1986.

HOLM, L.; YEO, R. The biology, control and utilization of aquatic weeds. Part I. Weeds Today, p. 7-13, 1980.
INSTITUTO BRASILEIRO DE MEIO AMBIENTE E DOS RECURSOS NATURAIS RENOVÁVEIS - IBAMA. In: WORKSHOP CONTROLE DE PLANTAS AQUÁTICAS, 1988, Brasília. Resumos... Brasília: IBAMA, 1998. p. 1-3.

MacDONALD, E. G.; LANGELAND, A.K. Aquatic weed manegement alternatives for tropical areas. In: CONGRESSO DE LA ASOCICION LATINOAMERICANA DE MALEZAS, 15, 2001, Maracaibo. Resumos...

Maracaibo: 2001. p. 44-47.

MARCONDES, D. A. S.; TANAKA, R. H. Plantas aquáticas nos reservatórios das usinas hidrelétricas da CESP. In: CONGRESSO BRASILEIRO DA CIÊNCIA DAS PLANTAS DANINHAS, 21, 1997, Caxambu. Workshorp de Plantas Aquáticas... Caxambu: Sociedade Brasileira da Ciência das Plantas Daninhas, 1997. p. 2-4.

MARTINS, D. et al. Controle químico de plantas daninhas aquáticas em condições controladas - caixas d'água. Planta Daninha, v. 17, n. 2, p. 289-96, 1999.

SOCIEDADE BRASILEIRA DA CIÊNCIA DAS PLANTAS DANINHAS - SBCPD. Procedimentos para instalação, avaliação e análise de experimentos com herbicidas. Londrina: 1995. $42 \mathrm{p}$. 